\title{
RECURSION OF SET TRAJECTORIES IN A TRANSFORMATION GROUP1
}

\author{
F. J. $\mathrm{HAHN}^{2}$
}

1. Introduction. In $[3]^{3}$ and [4] Trjitzinsky considers a one parameter group of homeomorphisms acting on a metric space. He considers an equivariant mapping which assigns to each point of the space a closed set which contains that point, and then introduces the notion of recurrence (stability). Here we consider a transformation group $(X, T, \pi)$, where $X$ is a metric space with the metric $d ; T$ is a topological group; and $\pi$ is a continuous mapping of $T \times X$ onto itself satisfying the following: If $e$ is the identity of $T$ then $\pi(e, x)=e x=x$, and if $t$ and $t^{\prime} \in T$ and $x \in X$ then $\pi\left(t, \pi\left(t^{\prime}, x\right)\right)=t\left(t^{\prime} x\right)=\left(t t^{\prime}\right) x=\pi\left(t t^{\prime}, x\right)$. The group $T$ is allowed to act on the subsets of $X$ and we discuss continuity, recursion, and a conjecture raised in [4, p. 99].

Notation. $A(X)=\{E \subset X: E \neq \varnothing\}, \quad B(X)=\{E \subset X: E \neq \varnothing, \bar{E}$ compact $\}, \quad K(X)=\{E \subset X: E \neq \varnothing$ and closed $\}, C(X)=\{E \subset X$ : $E \neq \varnothing$ and compact $\}$.

Definition 1.1. If $(X, d)$ is a metric space then the Hausdorff pseudo-metric $h$ on $A(X)$ induced by $d$ is given by

$$
h(D, E)=\max \left(h_{1}(D, E), h_{1}(E, D)\right)
$$

where

$$
h_{1}(D, E)=\sup \{d(x, E): x \in D\} .
$$

If $h$ is restricted to $K(X)$ or $C(X)$ then $h$ is a metric. The remainder of the discussion will concern itself primarily with $K(X)$ and $C(X)$. The results obtained may be applied to $A(X)$ and $B(X)$ after making the observation that $K(X)$ and $C(X)$ are homeomorphic to the quotient spaces obtained from $A(X)$ and $B(X)$ when one identifies a set with its closure.

DEFINITION 1.2. If $(X, T, \pi)$ is a transformation group then in the triple $(A(X), T, \pi)[(B(X), T, \pi),(K(X), T, \pi),(C(X), T, \pi)]$ we let $\pi(t, E)=t(E)=\{t x: x \in E\}$ for $E \in A(X)[B(X), K(X), C(X)]$.

2. Continuity. In the following sections we will always have in mind a transformation group $(X, T, \pi)$. If $t \in T$ then the $t$ transition of $X[K(X), C(X)]$ will be the mapping

Received by the editors September 18, 1959.

1 This paper is based on the author's thesis.

2 National Science Foundation Fellow.

3 References to the bibliography appear as numbers inside brackets. 


$$
\pi_{i}: X \rightarrow X[K(X) \rightarrow K(X), C(X) \rightarrow C(X)]
$$

defined by $\pi_{t}(x)=t x$ for $x \in X\left[\pi_{t}(E)=t(E)\right.$ for $E \in C(X)$ or $\left.K(X)\right]$.

THEOREM 2.1. If each t transition of $X$ is uniformly continuous then so is each $t$ transition of $K(X)$.

Proof. [2, p. 170].

Theorem 2.2. If $X$ and $T$ are locally compact then $(C(X), T, \pi)$ is a transformation group.

Proof. The homomorphism and identity properties are clear and we need only show that $\pi$ is continuous on $T \times C(X)$. Let $\left(t_{0}, E\right)$ $\epsilon T \times C(X)$ and let $\epsilon>0$ be arbitrary. Since $X$ is locally compact there is an $\eta>0$ such that $E(\eta)=\{x: d(x, E) \leqq \eta\}$ is compact and in $T$ there is a compact neighborhood $V$ of $e$. It follows that $t_{0} V \times E(\eta)$ is a compact neighborhood of $\left(t_{0}, E\right)$ in $T \times X$ and thus $\pi$ is uniformly continuous on this neighborhood. Consequently there is a $\delta>0$, and $<\eta$, and a neighborhood $U$ of $e, U \subset V$, such that if $t \in t_{0} U$, and $x$ and $y \in E(\eta)$, such that $d(x, y)<\delta$, then $d\left(t x, t_{0} y\right)<\epsilon / 2$. If $D \in C(X)$ is chosen so that $h(E, D)<\delta$ and $t \in t_{0} U$ then $h_{1}\left(t D, t_{0} E\right)$ $=\sup \left\{d\left(t x, t_{0} E\right): t x \in t D\right\}<\epsilon$ and similarly $h_{1}\left(t_{0} E, t D\right)<\epsilon$.

COROLlary 2.3. If $\pi$ is uniformly continuous on $T \times X$ then $(K(X), T, \pi)$ is a transformation group.

Theorem 2.4. If $\left(X, d^{\prime}\right)$ is a separable, locally compact, metric space and if $T$ is locally compact, then there is a metric $d$ equivalent to $d^{\prime}$ such that $(K(X), T, \pi)$ is a transformation group if $K(X)$ has the Hausdorff metric $h$ induced by $d$.

Proof. Since $X$ is locally compact and separable we can express $X$ as the countable union of compact sets. It follows that if $\hat{X}$ is the one point compactification of $X$ then $\hat{X}$ has a countable base and is thus metrizable by a metric $d$. The injection $i: X \rightarrow \hat{X}$ is a homeomorphism into $\hat{X}$ and thus $d$ is equivalent to $d^{\prime}$ and $X$ may be metrized by $d$. Let $h$ be the Hausdorff metric induced by $d$ on $K(X)$ and $K(\hat{X})$. Letting $t(\infty)=\infty$ for all $t \in T$ and applying Theorem 2.2 we see that $(K(\hat{X}), T, \pi)$ is a transformation group. We define the mapping $j: K(X) \rightarrow K(\hat{X})$ by $j(E)=\mathrm{Cl}\{i(E)\}=\mathrm{Cl}\{i(x): x \in E\}$ which indicates closure in $\hat{X}$. We see that $j$ is an isometry since

$$
h(D, E)=h(i(D), i(E))=h(\mathrm{Cl}\{i(D)\}, \operatorname{Cl}\{i(E)\})=h(j(D), j(E))
$$

and thus $(K(X), T, \pi)$ is a transformation group since $t j=j t$ for all $t \in T$ and $h(t E, t D)=h(t j(E), t j(D))$. 
Corollary 2.5. If $\left(X, d^{\prime}\right)$ is a locally compact, separable, metric space then there is a metric $d$ equivalent to $d^{\prime}$ such that each $t$ transition of $(K(X), h)$ is continuous where $h$ is the Hausdorff metric induced by $d$.

3. Recursion. Following Gottschalk and Hedlund [1, p. 21] we let $\mathfrak{U}$ be a distinguished class of subsets of $T$ which are called admissible.

Definition 3.1. $T$ is recursive at $E \in A(X)$ if for each $x \in E$ and each neighborhood $U$ of $x, U \subset X$, there is an admissible set $S \subset T$ such that $s E \cap U \neq \varnothing$ for each $s \in S$.

Definition 3.2. $T$ is regionally recursive at $E \in A(X)$ if for each $x \in E$ and each neighborhood $U$ of $x$ and $V$ of $E, U$ and $V \subset X$, there is an admissible set $S \subset T$ such that $s V \cap U \neq \varnothing$ for each $s \in S$.

We will say that $T$ is recursive [regionally recursive] on $A(X)$, $B(X), C(X)$ or $K(X)$ if $T$ is recursive [regionally recursive] at each point of $A(X)$ or $B(X), C(X)$ or $K(X)$ respectively. If in the above definitions $E$ reduces to a single point $\{x\}$ then the definitions are those given in $[1, \mathrm{p} .21]$. It is clear that $T$ is recursive [regionally recursive] on $X$ if and only if $T$ is recursive [regionally recursive] on $A(X)$.

For the remainder of the paper we let $\{P(i)\}, i \in I=\{1,2,3 \cdots\}$, be a sequence of subsets of $T$ and define an admissible set to be one which meets each $P(i)$.

THEOREM 3.1. If each $t$ transition of $C(X)$ is continuous and if $R$ is the set of $T$ recursive points of $C(X)$ then,

(a) $R$ is a $G_{\delta}$ subset of $C(X)$.

(b) If $T$ is regionally recursive on $X, R$ is a residual subset of $C(X)$.

Proof. If we let $E(n, m)$ be the collection of all $E \in C(X)$ for which there is an $x \in E$ such that $d(x, t E) \geqq 1 / m$ for all $t \in P(n)$, then $R=C(X)-U_{n, m=1} E(n, m)$. If $\left\{E_{j}\right\}, j \in I$, is a sequence in $E(n, m)$ such that $E_{j} \rightarrow E$ then in each $E_{j}$ we let $x_{j}$ be chosen so that $d\left(x_{j}, t E_{j}\right)$ $\geqq 1 / m$ for all $t \in P(n)$. For each $j \in I$ there is a $y_{j} \in E$ such that $d\left(x_{j}, y_{j}\right) \rightarrow 0$ and since $E$ is compact there is an infinite subset $I^{\prime} \subset I$ and a $y \in E$ such that if $k$ ranges over $I^{\prime}$ then $y_{k} \rightarrow y$ and consequently $x_{k} \rightarrow y$. If $E \notin E(n, m)$ then there is a $t \in P(n)$ for which $d(y, t E)$ $=1 / m-\eta$ where $\eta>0$. Since $t$ is continuous on $C(X)$ there is a $\delta>0$ such that if $h(E, D)<\delta$ then $h(t E, t D)<\eta / 4$ and there is a $k \in I^{\prime}$ such that $d\left(y, x_{k}\right)<\eta / 4$. It follows that

$$
\begin{aligned}
d\left(x_{k}, t E_{k}\right) & \leqq d\left(x_{k}, y\right)+d\left(y, t E_{k}\right) \\
& \leqq d\left(x_{k}, y\right)+d(y, t E)+\frac{\eta}{2} \leqq \frac{1}{m}-\frac{\eta}{4}<\frac{1}{m} .
\end{aligned}
$$


This is clearly impossible and it follows that each $E(n, m)$ is closed and (a) is proved.

Assume now that $T$ is regionally recursive on $X$. If $H(n, m)$ is the set of all $x \in X$ for which there is a $t \in P(n)$ such that $d(x, t x)<1 / m$ then each $H(n, m)$ is open and dense in $X[1, \mathrm{p} .25]$. If $\epsilon>0$ is arbitrary and if $E \in E(n, m)$ we cover $E$ with a finite number of spheres of radius $\epsilon$. In each such sphere there is an $x_{j} \in H(n, m)$. If $D=U x_{j}$ then $D$ is compact and $h(E, D)<\epsilon$ and $D \notin E(n, m)$. It follows that each $E(n, m)$ is nowhere dense and (b) is proved.

Lemma 3.2. If $U \subset X$ is a dense open set such that $X-U \neq \varnothing$ and if $\mathbb{E}=\{E: E \in K(X)$ and $d(E, X-U)>0\}$ then $\mathbb{E}$ is open and dense in $K(X)$.

Proof. Clearly $(\&$ is open. If $\epsilon>0$ is arbitrary and if $D \in K(X)$, then if we let $E=\{x: d(x, D)<\epsilon\}$ it suffices to show that $\bar{E} \in \overline{\mathbb{F}}$. Let $F=E \cap U$ and let $U(n)=\{x: d(x, X-F) \geqq 1 / n\}$. Since $U$ is dense and open we have $h(\bar{E}, F)=0$ and $F=\bigcup_{n \in I} U(n)$ and $U(n) \in \mathbb{E}$. It follows that $h(\bar{E}, U(n))=h(F, U(n)) \rightarrow 0$ as $n \rightarrow \infty$.

THEOREM 3.3. If each $t$ transition of $K(X)$ is continuous and if $T$ is regionally recursive on $X$ and if $R$ is the set of recursive points of $K(X)$ then $R$ is a residual subset of $K(X)$.

Proof. If $H(n, m)$ is defined as in Theorem 3.1 then each $H(n, m)$ is a dense open subset of $X$ and by Lemma 3.2 each $K(H(n, m))$ contains a dense open subset of $K(X)$. If $E \in \bigcap_{m, n=1} K(H(n, m))$ then $E \in R$ since if $x \in E$ and $U$ is a neighborhood of $x$ there is an $m$ such that $S(x, 1 / m) \subset U$ and thus for each $P(n)$ there is a $t_{n}$ such that $U \cap t_{n} E \neq \varnothing$. The set $\left\{t_{n}\right\}, n \in I$, is extensive. It follows that $R \supset \bigcap_{m, n=1} K(H(n, m))$ and so $R$ is a residual.

It is important to note that if $X$ is complete then so is $C(X)$ and $K(X)$ or if $X$ is locally compact then so is $C(X)$ [2, p. 161]. In these cases the residuals of $C(X)$ and $K(X)$ are dense.

4. Recursion and an equivariant mapping. In this section $\phi$ will be a continuous mapping of $X$ into $K(X)$ such that $x \in \phi(x)$ for all $x \in X$, and $t \phi=\phi t$ for all $t \in T$.

Definition 4.1. $\phi T$ is regionally recursive on $X$ if for each $x \in X$ and each neighborhood $U$ of $x$ there is an admissible set $S \subset T$ such that

$$
\bigcup_{y \in U} \phi(y) \cap s \bigcup_{y \in U} \phi(y) \neq \varnothing \quad \text { for all } s \in S .
$$

Definition 4.2. $\phi T$ is almost recursive at $x$ if for each neighbor- 
hood $U \supset \phi(x)$ there is an extensive set $S \subset T$ such that $U \cap s \phi(x) \neq \varnothing$ for all $s \in S$.

We remark that since $d(E, D) \leqq h(E, D)$ for $E, D \in A(X)$ it follows that $h(\phi(x), \phi(y))$ is simultaneously continuous in $x$ and $y$.

THEOREM 4.3. If $R \subset X$ is the set of almost recursive points then

(a) $R$ is a $G_{\delta}$ subset of $X$.

(b) If $\phi T$ is regionally recursive then $R$ is a residual subset of $X$.

Proof. If $E(n, m)$ is the set of all $x \in X$ such that $d(\phi(x), t \phi(x))$ $\geqq 1 / m$ for all $t \in P(n)$ then $E(n, m)$ is closed since if a sequence $\left\{x_{j}\right\}$ of $E(n, m)$ converges to $x$ then $d\left(\phi\left(x_{j}\right), t \phi\left(x_{j}\right)\right)$ converges to $d(\phi(x), t \phi(x))$ for $t \in T$. If there is an $E(n, m)$ which is somewhere dense then there is an $\epsilon>0,0<\epsilon<1 / m$, and a $y \in E(n, m)$ such that the $\epsilon$ sphere about $y, S(y, \epsilon) \subset E(n, m)$. Choose $\delta>0$ and $<\epsilon$ such that if $x \in S(y, \delta)$ then $h(\phi(x), \phi(y))<\epsilon / 2$. Since $\phi T$ is regionally recursive there is a $t \in P(n)$ such that $\bigcup_{z \in S(y, \delta)} \phi(z) \cap t \bigcup_{z \in S(y, \delta)} \phi(z) \neq \varnothing$. It follows that there are $z$ and $w \in S(y, \delta)$ for which $\phi(z) \cap t \phi(w) \neq \varnothing$ and $h(\phi(z), \phi(w)) \leqq h(\phi(z), \phi(y))+h(\phi(y), \phi(w))<\epsilon$. If $u \in \phi(z) \cap t \phi(w)$ then

$$
\begin{aligned}
d(\phi(w), t \phi(w)) \leqq d(u, \phi(w)) & \leqq \sup \{d(v, \phi(w)): v \in \phi(z)\} \\
& \leqq h(\phi(z), \phi(w))<\epsilon<\frac{1}{m}
\end{aligned}
$$

and therefore $w \notin E(n, m)$ but this contradicts $w \in S(y, \delta) \subset E(n, m)$ and thus $E(n, m)$ is nowhere dense. Since $R=X-\bigcup_{n, m=1} E(n, m)$ our conclusions follow.

In $[4$, p. 99] Trjitzinsky considered $T$ to be a one parameter group and defined $L^{+}(\phi(x))$ to be the set of all points $y \in X$ such that $\lim \inf _{t \rightarrow+\infty} d(y, t \phi(y))=0$. He conjectured that if for all $x$ in a dense subset of $X$ we have $L^{+}(\phi(x)) \supset \phi(x)$ then there is a residual such that for all $x$ in this set $\lim \inf _{t \rightarrow+\infty} d(x, t \phi(x))=\lim \inf _{t \rightarrow-\infty} d(x, t \phi(x))=0$. If we let $P(i)=(i,+\infty)$ and $P(-i)=(-\infty,-i)$ for $i \in I$ then Theorem 4.2 proves this conjecture.

If the fact that $x \in \phi(x)$ is dropped then Trjitzinsky's conjecture is false because $\phi T$ may not be regionally recursive. Consider the following example. Let $Z=C \times C$ where $C$ is the unit circle. Let $(Z, T, \Gamma)$ be the transformation group given by the solution to the differential equations

$$
\frac{d x}{d t}=a\left(x^{2}+y^{2}\right), \quad \frac{d v}{d t}=b\left(x^{2}+y^{2}\right),
$$


where $x$ and $y$ are coordinates modulo one and $a / b$ is irrational. There is a point $z \in Z$ such that $T z$ is Poisson stable $(+)$ but not $(-)$. The set of points which are Poisson stable $(+)$ and $(-)$ are dense in $Z$.

In $E_{3}$ with coordinates $(\xi, \eta, \tau)$ consider the cylinder

$$
Y=\left\{y: y=(\xi, \eta, \tau), \xi^{2}+\eta^{2}=1\right\} .
$$

Define the transformation group $(Y, T, \Lambda)$ as follows:

$$
\Lambda(t, y)=t(y)=t(\xi, \eta, \tau)=(\xi, \eta, \tau+t) .
$$

Imbed $Z$ in $E_{3}$ such that $Z \cap Y=\varnothing$ and let $X=Z \cup Y$. We now define $(X, T, \pi)$ and $\phi: X \rightarrow C(X)$ as follows:

$$
\begin{array}{rrr}
\pi(t, x) & =\Gamma(t, x) & \text { for } x \in Z, \\
\pi(t, x) & =\Lambda(t, x) & \text { for } x \in Y, \\
\phi(x) & =x & \text { for } x \in Z, \\
\phi(x) & =\pi(\tau, z)=\tau(z) & \text { for } x \in Y \text { and } x=(\xi, \eta, \tau) .
\end{array}
$$

All the conditions of Trjitzinsky's conjecture are satisfied except $x \in \phi(x)$ and for each $x \in Y$ we have lim inf $t_{t \rightarrow-\infty} d(t \phi(x), \phi(x)) \neq 0$ and $Y$ is category II in $X$.

The hope that the conclusion of Theorem 4.2 can be strengthened to read "then $\phi T$ is recursive on a residual" is in vain. Let $X=E_{2}$ with coordinates $x=\left(x_{1}, x_{2}\right)$ and define $(X, T, \pi)$ for $T$ the additive group of the reals as follows: $\pi(t, x)=\left(x_{1} e^{t}, x_{2} e^{t}\right)$. If we define $\phi(x)$ $=\left\{y: y_{1}^{2}+y_{2}^{2} \leqq x_{1}^{2}+x_{2}^{2}\right\}$ then $\phi T$ is regionally recursive on $X$, where an admissible set is one that meets each open infinite interval, but for $x \neq(0,0)$ we have $L^{-}(\phi(x)) \subset \phi(x)$ properly.

\section{BIBLIOGRAPHY}

1. W. Gottschalk and G. Hedlund, Topological dynamics, Providence, Amer. Math. Soc. Colloquium Publications, vol. 36, 1955.

2. E. Michael, Topologies on spaces of subsets, Trans. Amer. Math. Soc. vol. 71 (1951) pp. 152-183.

3. W. Trjitzinsky, Problèmes dans la théorie des systèmes dynamiques, Acta Math. vol. 95 (1956) pp. 191-289.

4. - Aspects topologiques de la théorie des fonctions réelles et quelques conséquences dynamiques, Ann. Math. Pura Appl. Ser. 4 vol. 42 (1956) pp. 51-117.

UNIVERSITY OF ILLINOIS 